\title{
Evaluation of Heuristic Algorithms for Scheduling, Routing and Power Allocation in Traffic Sensitive Spatial TDMA Wireless Ad Hoc Networks
}

\author{
Oscar Somarriba \\ Department of Communication Systems \\ The Royal Institute of Technology (KTH) \\ Electrum 418, S-164-40 Kista, Sweden \\ oscarseradio.kth.se
}

\begin{abstract}
Major design issues in Wireless Ad Hoc Networks is the design of the routing algorithms and the Medium Access Control (MAC) protocols. Spatial TDMA (STDMA) is a "conflict-free" MAC protocol for these networks, thus enabling high spectral utilization. In link-oriented STDMA, the schedule specifies when particular radio links will be activated. Moreover, the use of power control and heuristic routing algorithms have the potential of improving STDMA network performance. Besides Power and Traffic Adaptive STDMA, in this paper we also, as a comparison, include optimal STDMA. To obtain the optimal STDMA scheduling combined with routing and power allocation, a convex optimization problem is solved using the method reported in [1]. Our results show that one of the heuristic routing algorithms can provide near optimal solutions regarding end-toend network throughput.
\end{abstract}

\section{INTRODUCTION}

Multi-hop Wireless Networks (MWN), are a collection of radio units (nodes) forming a (temporary) network without the use of any existing infrastructure or centralized administration. MWN must support a wide category of services. The different services all have different quality of service (QoS) demands. Quick adaptation to changes in the environment can be achieved at the lower layers in the OSI model [1],[2].

These networks usually carry packets of data between nodes equipped with radio transceivers and omnidirectional antennas. In many MWN, not all packet radio nodes can communicate directly because of interference, range limitations, or natural obstacles. In this situation, a packet transferred between two distant nodes may have to be relayed by intermediate stations or nodes. Major design issues in these networks involve the "path finding" methods, i.e. routing algorithms, and the selection of the Medium Access Control (MAC) protocols that determine how nodes share the channel to transmit their packets. So, on the one hand, each node must perform routing tasks, i.e., selection of a set of intermediate links (path) between each source $(S)$ and the final destination (D) of packet (denoted by an (S,D) pair). Besides, routing also influences the Multiple Access Interference (MAI) created by sharing the radio channel thus limiting the potential throughput. This problem can be the result of limited network connectivity but can also be due to poor route selection that may arise when more than one path exits between an (S,D) pair. On the other hand, we study here "conflict-free" multiple schemes, which have been proposed to ensure that a packet transmission, whenever made, is successful. One of the "conflict-free" MAC protocols for MWN is Spatial TDMA (STDMA) [3]. When using STDMA as the MAC protocol, a link transmission schedule is created in advance. So, STDMA defines a repeating transmission schedule (frame) that contains a fixed number of slots with each slot being assigned to a unique set of non-conflicting links (compatible links). We will denote by $\mathbf{S}$ the STDMA schedule of an MWN. Furthermore, scheduling algorithms are important components in providing guarantees quality of service parameters such as delay or throughput. Thus, the design of STDMA algorithms has received considerable attention in literature [4],[5],[6],[7].

The STDMA schedule algorithms adapted to traffic patterns are commonly referred as Traffic Controlled STDMA (TC STDMA) or Traffic Sensitive STDMA. Moreover, previous results [6] show that controlling transmitters' power in wireless communication networks provides numerous benefits. It allows for efficient sharing of the same radio channel to achieved required quality of service levels, minimizing the power spent in the process. To further improve network performance the impact of combined routing and scheduling for TC STDMA has been proposed in [8]. In this paper, we extend those results by incorporating power allocation instead of adaptive antennas. Complementary, the problem of joint scheduling, routing, and power allocation has been formulated in ([1],[9]) as a crosslayer optimization problem.

In this study, we consider how network capacity, in terms of throughput, is affected by nodes that are capable of adjusting transmit power in an STDMA network. We compare heuristic strategies with jointly (near) optimal end-to-end communication rates, routing, power allocation and transmission scheduling. To obtain the optimal scheduling combined with routing and power allocation, a convex optimization problem is solved using the method reported in [1]. In this way we compare Traffic sensitive STDMA (a situation where the traffic is known) protocols and the "optimal" STDMA. This allows us to gain insight in the influence of power control, spatial 
reuse, and routing strategies on the network performance, and provides a benchmark for evaluating heuristic strategies.

The organization of the paper is as follows. The network modeling of WMN are first presented in the next section, while the optimization formulation and the heuristic strategies, are both detailed in section III. Numerical results of optimal STDMA and heuristic approaches are shown in section IV. The article concludes with final remarks in section V.

\section{System MOdeL}

We assume that an MWN is formed by a set of $N$ nodes equipped with radio transceivers and isotropic antennas.

\section{A. Link Quality and Connectivity (Physical Layer)}

We represent the topology of the network by a directed graph, with nodes labeled $n=1, \ldots, N$ and with links labeled $l=1, \ldots, M(\mathrm{M} \leq \mathrm{N}(\mathrm{N}-1))$. The propagation effect is modeled by the radio propagation losses. Let $G_{i j}$ denote the power gain between transmitter node $i$ and the receiver node $j$. Usually, all the possible gains in a MWN are collected in the so-called gain matrix $\mathbf{G}=\left\{G_{i j}\right\}$. We use a deterministic fading model $G_{i j}=\left(d_{i j}\right)^{-\alpha}$. Here $d_{i j}$ is the distance between node $i$ and node $j$, and $\alpha$ is the path loss exponent. Furthermore, the Signal-to-Interference-Noise Ratio (SINR) is used as a measure of the link quality. For a link $(i, j)$ we introduce the SINR, $\Gamma_{i j}$, when node $i$ transmits with power $P_{i}$, to node $j$. In order to have a reliable link $(i, j)$ a minimum SINR is required, referred to as the SINR threshold $\gamma_{0}$. In STDMA, Multiple Access Interference (MAI) is controlled by scheduling compatible links. Thus, a set of links $\mathcal{L}$ can be activated if

$$
\Gamma_{i j}=\frac{P_{i} G_{i j}}{P_{\text {Noise }}+\sum_{k \neq i, j} G_{k j} X_{k} P_{k}} \geq \gamma_{0} \quad \forall \operatorname{link}(i, j),(k, j) \in \mathcal{L}
$$

Here we have introduced the binary variable whose entries $X_{k}$ are defined via $X_{k}=1$ if node k transmits, and $X_{k}=$ 0 otherwise. Besides, $P_{\text {Noise }}$ is the background noise power level at receiving node $j$ and $P_{k}$, the transmitter power of the node $k$. On the other hand, if packets can be successfully transmitted between two nodes while there is no interference from any other node then those two nodes are connected, and we say that this is a feasible or usable link. The study was confined to connected networks, i.e., networks where every node can be reached from another one with a finite number of hops. Connectivity $(\mathcal{C})$ is defined as the fraction of nodes in the network that can be reached by a node in one hop on average, i.e. $(\mathrm{M} / \mathrm{N}(\mathrm{N}-1))$, where $\mathrm{M}$ is the number of directed (unidirectional) radio links in the network. A sample 10-nodes network is shown in Figure 1.

\section{B. Data Link Layer}

High STDMA system capacities can be achieved by means of incorporating traffic load measurements into the MAC protocol design; again these schemes are commonly named

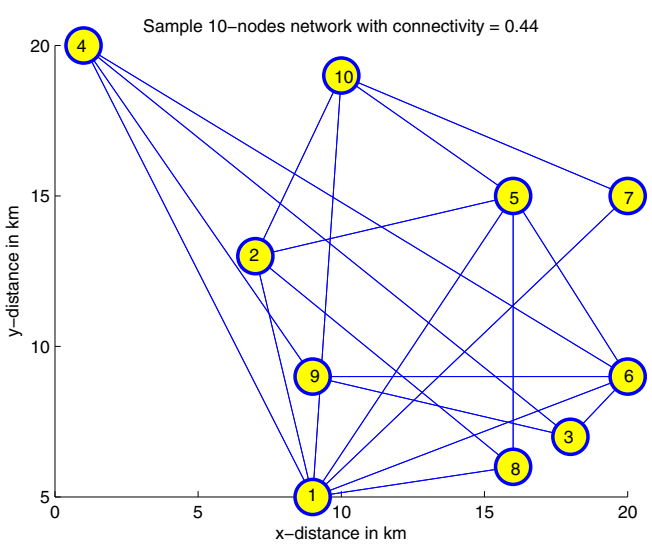

Fig. 1. Sample network with $\mathcal{C}=0.44$ in a given area of $20 \times 20 \mathrm{~km}^{2}$. The solid lines represent the connections among the nodes.

as Traffic Controlled STDMA (TC STDMA) protocols [5]. For the cross-layer design, two major schemes exist in this category: directional antenna-based MACs and MACs with power control. Also, we will partially focus our study on the latter issue. Because the application of power and rate control have the potential of significant improving of the network performance. So, STDMA protocols that takes advantage of multirate power control are introduced in Ref. [10]. In particular, among others, we will consider TC STDMA with variable transmitter power.

\section{Traffic}

The traffic from each node consisted of packets generated according to the Poisson distribution. The packet destinations were randomly distributed over all other nodes in the MWN with equal likelihood, so the traffic load on each link was topology dependent. If we label the set of source to destination traffic pair (S, D) by $p=1, \ldots, N(N-1)$ the average external traffic load transport by the network is given by

$$
\lambda=\sum_{S=1}^{N} \sum_{\forall D \neq S} \lambda_{D}^{S}=\sum_{p=1}^{N(N-1)} \lambda_{p}
$$

where $\lambda_{p}$ denotes the average traffic load between source-todestination pair $p$. We use the term end-to-end rates when $\lambda_{p}$ is given in bits/s and traffic load when is measure in packets/timeslot or packets/slot. Due to a store-and-forward mechanism, packets between (S, D) pairs may travel through intermediate nodes. Therefore, the average traffic load going through a link $l$ is the result of external and internal traffic [4] and it is given by eqn. (3):

$$
\Pi_{l}=\sum_{p=1}^{N(N-1)} r_{l p} \lambda_{p}
$$

where $\Pi_{l}$ are the elements of the link traffic vector $\Pi=\left[\begin{array}{llll}\Pi_{1} & \Pi_{2} & \ldots & \Pi_{L}\end{array}\right]^{T}$. Here we have also introduced the linkroute incidence matrix $R \in \mathbf{R}^{L \times N(N-1)}$ whose entries $r_{l p}$ are defined via $r_{l p}=1$ if the traffic between node pair $p$ is routed across link $l$, and $r_{l p}=0$ otherwise. By denoting the vector of source to destination traffic load $\Lambda=\left[\begin{array}{llll}\lambda_{1} & \lambda_{2} & \ldots & \lambda_{N(N-1)}\end{array}\right]^{T}$, then the eqn. (3) can be written in matrix form as $\Pi=\mathbf{R} \Lambda$. 


\section{Network Layer: Routing Algorithms}

We will consider the following particular classes of routing schemes.

1) Free routing: In this assignment [1], each node can send data to many destinations and receive data from many sources, but multicast is not considered. This routing scheme can be taken as a reference (benchmark) for the forthcoming heuristic schemes.

2) Minimum Hop Algorithm (MHA): We use the minimum cost routing where cost metric for all links are equal to one. The minimum cost routing problem is here solved with Dijkstras algorithm [11]. This creates a routing table that minimizes the number of hops needed to transport a packet to its destination node. So, the routing scheme used here is termed the Minimum Hop Routing Algorithm (MHA).

3) ARA-MHA (Advanced Reuse Adaptive-MHA): This integrated routing assignment is a modification of the one introduced in [8], in this case it combines scheduling and routing with SINR balancing power control [12]. In addition to that, omnidirectional antennas are utilized instead of directional antennas as was originally proposed.

4) BMPRA (Balanced Minimum Power path Routing Algorithm): This is a variant assignment policy of MPRA (Minimum Power path Routing Algorithm) where we re-route bottleneck paths as it is described in [12]. MPRA is an assignment policy where routing is a function of distances between nodes. In short, MPRA will try to route the traffic in such a way as to minimize the transmitter power in the involved paths that support communication.

\section{E. Performance Measures}

To evaluate the network performance, we use two performances measures; namely the end-to-end packet delay and the end-to-end throughput. The end-to-end packet delay is defined as the time between the arrival of a packet at the buffer of the originating node and the end of the slot in which it is successfully received at the final destination. Moreover, an important performance measure is also the end-to-end throughput, which is number of packets successfully transported through the network per time unit. We are particular interested in the maximum end-to-end throughput, which can be defined as the largest input traffic $\lambda^{*}$ giving bounded (finite) end-to-end packet delay. In this work we study some heuristic strategies for scheduling, routing and power allocation in MWN, with emphasis on max-min fair allocation of network resources. That motivation comes from the fact we have experienced that throughput maximization solutions usually tend to activate a few links and allocates non-zeros rates to the flows that only transverse these links. All other flows are set to zero.

\section{STDMA SCHEDULING}

Defining $\mathbf{S}=\left[\begin{array}{llll}s_{1} & s_{2} & \ldots & s_{T_{f}}\end{array}\right]$ where $s_{i}$ are the column vectors of $\mathbf{S}$ which hold the data rates of $L$ feasible links, and $T_{f}$ is the duration of the STDMA schedule in timeslots. Besides, if $\alpha=\left[\begin{array}{lll}\alpha_{1} & \ldots & \alpha_{T_{f}}\end{array}\right]^{T}$ is the vector of weights corresponding to the fraction of time each column in $\mathbf{S}$ is activated within a
STDMA frame. The link capacity is represented by the vector $\mathbf{c}=\left[\begin{array}{lll}c_{1} & \ldots & c_{L}\end{array}\right]^{T}$. The link capacity allocated by the scheduling is given by [1], [10]:

$$
\mathbf{c}=\mathbf{S} \alpha ; \quad \sum_{k=1}^{T_{f}} \alpha_{k}=1
$$

In general, the capacity allocation can end up maximizing an objective function $u$ (e.g. [10]). Let $u(\cdot)$ be an utility function of the end-to-end rates $\Lambda$. Re-writing eqn. (7) from [1] (referred as the restricted master program) and using eqn. (4), the optimization problem can be formulated as follows

$$
\begin{array}{ll}
\operatorname{maximize} & u(\Lambda) \\
\text { subject to } & \mathbf{R} \Lambda \preceq \mathbf{S} \alpha, \quad \Lambda \succeq \mathbf{0}, \quad \alpha \succeq 0 \\
& \sum_{k=1}^{K} \alpha_{k}=1
\end{array}
$$

This is similar to the formulation used for investigating the capacity of a number of small MWN in [10]. In general, this allocation is an NP-complete problem since $\mathbf{S}$ grow exponentially with the number of links. However, for relative small networks, there exist some methods to find the (near) optimal solution [1], [7].

\section{A. The Convex Optimization Approach for Scheduling}

We use the convex optimization approach reported in [1] to tackle the capacity allocation problem formulated in (5) and apply the column generation method to find the column vector of $\mathbf{S}$ that maximize the minimum source to destination or end-to-end rates. An important design problem for MWN is the so-called max-min fairness. An allocation $\Lambda$ is called max-min fair if an increase in any component $\lambda_{i}$ of $\Lambda$ must cause a decrease in an already smaller component. The set of source to destination rates can be found by solving the following problem

$$
\begin{array}{ll}
\operatorname{maximize} & \lambda_{\min } \\
\text { subject to } & \lambda_{\min } \leq \lambda_{p}, \quad \forall p . \\
& \text { and the constraints in (5) }
\end{array}
$$

where $\lambda_{\min }$ is the minimum end-to-end rate. Consequently the bound for the end-to-end throughput (in Packets/timeslot) that produces a finite average end-to-end packet delay can be expressed as

$$
\lambda_{o p t}^{*}=N(N-1) \lambda_{m i n} / R_{b}
$$

where $R_{b}$ is the base rate. Here we are interested in a particular max-min fair solution, the so-called maximum equalrate allocation (sometimes called the uniform capacity [5]) where we seek the maximum end-to-end rate that can be sustained by all source-destination pairs simultaneously. The maximum equal-rate in the SRRAS (simultaneous routing, resource and scheduling) approach [1] is obtained by replacing the inequalities $\lambda_{\text {min }} \leq \lambda_{p}$ in the max-min fair formulation by equalities, i.e., $\lambda_{\min }=\lambda_{p}$. In summary, we assure the same $\lambda_{p}=\lambda^{\text {equal }}=\lambda^{*} /(N)(N-1)$ for all source-destination pairs. 


\section{B. Heuristics for Maximum Minimun throughput}

Since, optimization methods are not practical to utilize in real (large) networks, heuristic algorithms may be an interesting alternative to design MWN (this may end up improving computation at the price of relative modest performances losses). Here, we consider the following two TC STDMA protocols:

Scheme CR: Fixed Transmission rates and maximum power transmissions. In this model, transmitters send with maximum power or stay silent. A collection of links can be active in the same time slot only if all active links exceed their SINR targets (c.f. [7]). We have used a similar approach to the heuristic proposed in [5]. If the source to destination traffic load are set to be equal, this algorithm target to do max-min fair allocation. For fixed transmission rates we use $R_{b}$. CR stands for Constant Transmission Rate.

Scheme PC: Fixed Transmission rates and SINR balancing. In this scheme, transmitters of active links use the minimum power necessary to reach their SINR target (c.f. [6], [10]). Compared to the Scheme I, this approach saves energy, creates less interference, and has the potential to increase spatial reuse. We have used a similar approach to the Scheme CR extended to utilize SINR balancing power control. PC stands for Power Control.

For the above Schemes, the bounded end-to-end throughput (in Packets/timeslot) is given by [5]

$$
\lambda_{h}^{*}=N(N-1) \lambda_{\min } / R_{b}=N(N-1) \min _{\forall l}\left(\frac{n_{l}}{T_{f} \Pi_{l}}\right)
$$

where $n_{l}$ is the number of slots within a period of the heuristic schedule, $T_{f}$, allocated to link $l$.

\section{NUMERICAL EXAMPLES}

To evaluate our methodology, we constructed a set of 80 sample networks composed by 10 nodes as described in section II using the frequency of $300 \mathrm{MHz}$. We let $\alpha$ $=3$, Pnoise $=1.2655 \times 10^{-13}$ Watts. To generate the network topology, we place nodes randomly within a given area $\left(20 \times 20 \mathrm{~km}^{2}\right)$ and introduce links between every pair of nodes that can sustain the base target SINR $\left(\gamma_{o}=10\right)$ when all other transmitters are silent. To do so we adjust the transmit range progressively so that the nodes form a network that is fully connected and that $(M / N(N-1))$ matches a desired target number (target connectivity). Thus, we reach $P_{\max }=\gamma_{o} P_{\text {noise }} / g^{\text {min }}$, where $g^{\text {min }}=\min \mathbf{G}$ over all the feasible links of a particular network realization. We also use the Shannon capacity formula $c_{\text {tgt }}^{(r)}=W \log _{2}\left(1+\gamma_{\text {tgt }}^{(r)}\right)$ to relate target SINR-levels to rates. Here, $c_{\mathrm{tgt}}^{(r)}$ and $\gamma_{\mathrm{tgt}}^{(r)}$ denote the $r^{\text {th }}$ discrete rate level and the associated SINR target, respectively. Using $W=1 \mathrm{MHz}$ and a SINR-target of $\gamma_{\mathrm{tgt}}^{R_{b}}=10$, we find the base rate $\left(R_{b}\right) 3.49$ Mbps.

First of all, we consider the impact of the routing assignment on the network performance. For instance, we can utilize the 10-nodes network shown in Figure 1 and then, we compute for it the network behavior according to some of the routing schemes described in subsection II-D, but free routing is not

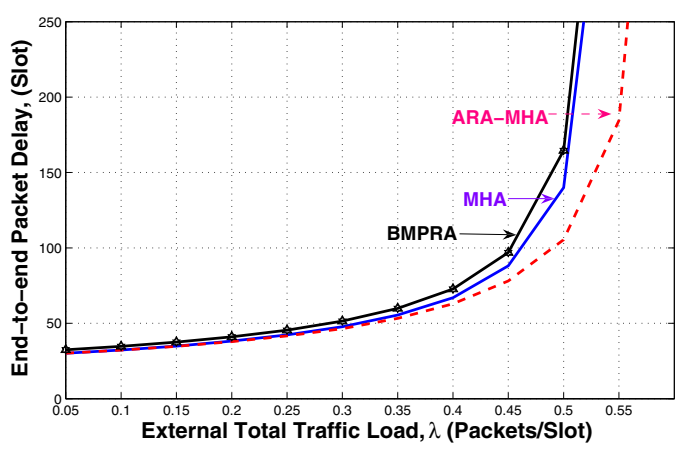

Fig. 2. Packet delay vs. External traffic load. MAC protocol: TC STDMA. Network of 10 nodes shown in Figure 1. Routing assignments: BMPRA, MHA, and ARA-MHA.

yet studied. The resulting delay-throughput operating curves are determined via network simulations and these are shown in Figure 2. Similar results have been obtained for 20-nodes networks.

General speaking, we can observe in Figure 2 that the maximum throughput with BMPRA (first curve to the left) is slightly poor in comparison with the other assignments. This is due to the fact that BMPRA [12] implies the use of "minimum distance" as well as some load balancing of the bottleneck traffic. Besides, MHA tends to concentrate traffic in some links which are "well-located" relative to other nodes, ending up sometimes with a saturation or bottleneck situation. Moreover, ARA-MHA outperforms the other routing assignments since the use of SINR balancing power control with RA-MHA [8] implies significant reduction of interference. Since BMPRA is the worst-cases in comparison with the other routing schemes, consequently we will refrain to continue studying it, here.

Let us turn now to the free routing scheme and the convex optimization approach for the scheduling of the two types of STDMA protocols described in III-B. In other words, in order to be able to make comparisons, we first study the analogous cases presented in subsection III-B for the convex optimization approach (optimal STDMA) with the maximun equal-rate allocation we compute $\lambda_{\text {opt }}^{*}$. Afterwards, we then compute the heuristic schedule $\left(S_{h}\right)$ and by applying eqn. (7) we end up with $\lambda_{h}^{*}$.

For easy analysis and make comparisons of the results per STDMA protocol (i.e., Scheme CR or Scheme PC), one can resort to compute the mse (mean square error) taking as a benchmark, the outcomes yielded by optimal STDMA jointly with the free routing scheme.

It is worthwhile to explain the legends attached to the Figures 3 and 4 (average end-to-end network throughput as a function of the connectivity). For the optimal STDMA combined with free routing (FR), we named the resulting curves, "Optimal Throughput for free routing". On the other hand, for the cases of TC STDMA with ARA-MHA and MHA algorithms, we termed the curves as follow: "Heuristic Throughput with ARA-MHA", and "Heuristic Throughput with $M H A$ ", respectively.

Let us begin with the Scheme CR. In Figure 3, we can observe that optimal STDMA combined with FR has slightly 


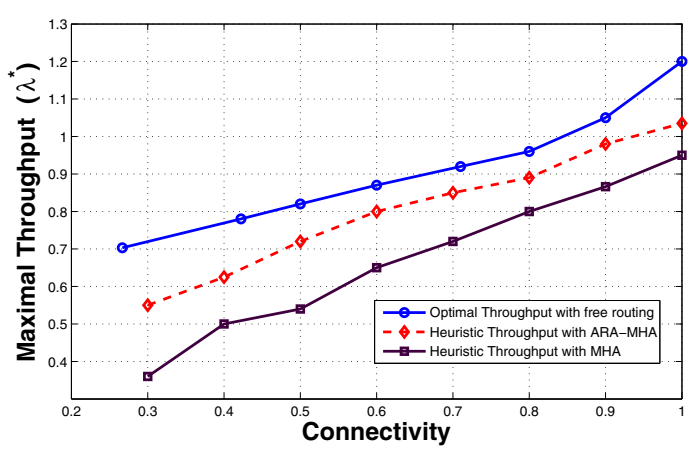

Fig. 3. Performance comparison for the average end-to-end network throughput (in Packets/timeslot) versus connectivity. Scheme CR.

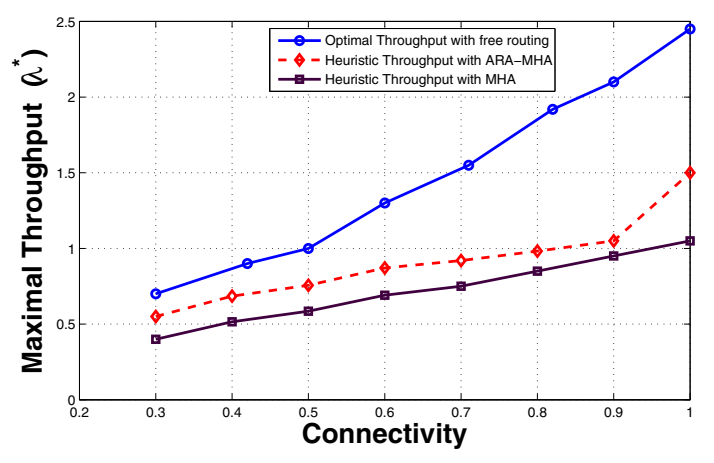

Fig. 4. Performance comparison for the average end-to-end network throughput (in Packets/timeslot) versus connectivity. Scheme PC.

better performance than TC STDMA (Maximum Transmitter Power) with ARA-MHA routing. The mse for this case equals to 0.013. Moreover, for the case of the Scheme $\mathbf{C R}$ with the MHA algorithm, the mse is equal to 0.06. Clearly, the throughput of ARA-MHA outperforms the throughput of the MHA algorithm. Of course, we can also end up with nodes that are transmitting at maximum power, which usually may increase the interference experienced by them, thus producing less spectrum spatial reuse factor in comparison with the Scheme PC.

For the Scheme PC in Figure 4, we can observe that the optimal STDMA protocol has a significant better performance than TC STDMA with SINR balancing Power control combined with the ARA-MHA routing. For this case the mse is equal to 0.4624 . Besides, when we have MHA as a routing assignment the situation is even worst, and the mse equals to 0.7816. Therefore, the Scheme PC provides more substantial spectrum spatial reuse factor thus increasing the end-to-end network throughput.

\section{CONCLUSIONS}

We have considered the problem of finding the optimal end-to-end rate selection, routing and power allocation, and transmission scheduling for STDMA in MWN. Our objective function has been the max-min fairness end-to-end network throughput. To do so, we have applied a specialized solution method based on column generation technique [1] to several numerical examples to compute the optimal STDMA jointly with free routing. Then, this is will be used as a benchmark platform. In addition to that, we have studied how close are Scheme CR and Scheme PC combined with heuristic routing approaches (ARA-MHA and MHA) to the optimal case (including FR).

For Scheme CR, the differences among optimal STDMA combined with FR and heuristic routing approaches is estimated by mse, which lies is the range of $1.3-6 \%$.

Complementary, for Scheme PC, the differences among optimal STDMA utilizing free routing and TC STDMA combined with heuristic routing can be estimated as mse values in the interval of $46-78 \%$. We note that as the network can now utilize more spatial reuse to maintain multiple active transmissions, and at any time instant it is possible that more than one streams are serviced. Of course, this is fully exploited by optimal STDMA jointly with free routing as it can be observed in Figure 4.

In the Scheme CR, the utilization of the routing assignment ARA-MHA can provide a near-optimal network capacity (in terms of throughput), and it could provide a good guidance in the design of MWN, when we consider the results above detailed.

\section{REFERENCES}

[1] M. Johansson and L. Xiao, "Cross-layer optimization of wireless networks using nonlinear column generation," IEEE Transactions on Wireless Communications, vol. 5(2), pp. 435-445, February 2006, to appear.

[2] L. Farman, U. Sterner, and O. Tronarp, "Analysis of capacity in ad hoc networks with variable data rates," in IEEE VTC-Spring, Milan, Italy, May 2004, pp. 2101-2105.

[3] R. Nelson and L. Kleinrock, "Spatial-tdma, a collision-free multihop channel access protocol," IEEE Transactions on Communication, vol. 33, no. 9, pp. 934-944, September 1985.

[4] J. Shor and T. Robertazzi, "Traffic sensitive algorithms and performance measures for the generation of self-organizing radio network schedules," IEEE Transactions on Communication, vol. 41, no. 1, pp. 16-21, January 1993.

[5] J. Grönkvist, "Interference-based scheduling in spatial reuse tdma," Department of Signals, Sensors and Systems, KTH, Stockholm, Sweden, Tech. Rep., September 2005.

[6] O. Somarriba and T. C. Giles, "Transmission power control for spatial TDMA in wireless radio networks," in Proceedings of the 4th IEEE Conference on Mobile and Wireless Communication Networks, Stockholm, Sweden, September 2002.

[7] P. Värbrand, D. Yuan, and P. Björklund, "Resource optimization of spatial TDMA in ad hoc radio networks: A column generation approach," in IEEE Infocom, San Francisco, CA, April 2003.

[8] M. Sanchez, T. C. Giles, and J. Zander, "Combined scheduling and routing for spatial tdma in multi-hop ad hoc networks," in Proceedings of the IEEE 2002 International Symposium on Wireless Personal Multimedia Communications, Honolulu, Hawaii, USA, September 2002.

[9] R. L. Cruz and A. Santhanam, "Optimal routing, link scheduling and power control in multi-hop wireless networks," in IEEE Infocom, San Francisco, CA, April 2003.

[10] S. Toumpis and A. Goldsmith, "Capacity regions for wireless ad hoc networks," in IEEE International Conference on Communications, New York, NY, May 2002.

[11] D. Bertsekas and R. G. Gallager, Data Networks. Prentice Hall, 1991.

[12] O. Somarriba, "Heuristic algorithms for scheduling and routing in spatial tdma wireless ad hoc networks," in Proceedings of the 4th Scandinavian Workshop on Wireless Ad Hoc Networks 2004, Stockholm, Sweden, May 2004. 